\title{
Early intervention likely improves mortality in COVID-19 infection
}

\author{
Authors: Daniel K Goyal, ${ }^{\mathrm{A}}$ Fatma Mansab, ${ }^{\mathrm{B}}$ Amir Iqbal ${ }^{\mathrm{C}}$ and Sohail Bhatti ${ }^{\mathrm{D}}$
}

\begin{abstract}
While some risk factors have been identified, the reasons for the disparities in disease progression with COVID-19 are unclear, with some patients developing progressive and severe disease while in others the course is benign. Given this sense of randomness, and in the absence of a definitive treatment, medical professionals can feel helpless. It is useful to remember how much can be done to affect the trajectory of illness, even without a 'magic bullet'. With evidence emerging that late presentation is directly associated with increased mortality, we make the case for increased vigilance in the community and earlier intervention.
\end{abstract}

KEYWORDS: COVID-19, SARS-CoV-2, intervention, treatment, oxygen

DOI: $10.7861 /$ clinmed.2020-0214

\section{Introduction}

On the face of it, the progression to acute respiratory distress syndrome (ARDS) in patients with COVID-19 seems almost random. Some contract COVID-19 and have a benign illness. Others have a progressive illness with varying degrees of viral pneumonia, pneumonitis and ARDS.?

As yet the reason for this disparity remains unknown. Age appears to be an independent risk factor, as well as probably ischaemic heart disease and diabetes, but anyone of any age can develop progressive and severe COVID-19 disease. 1 Within this sense of randomness, and in the absence of a clear and definitive pharmaceutical treatment to halt the disease and prevent progression, there is a genuine concern that medical professionals feel helpless and take the ill-informed view that there is little else that can be done. ${ }^{2}$

Authors: ${ }^{A}$ clinical coordinator, COVID-19 Team, Public Health Gibraltar and Internal Medicine, Department of Medicine, St Bernard's Hospital, Gibraltar Health Authority; Bresearch lead, COVID 19 Team, Public Health Gibraltar, Gibralter, UK; 'general practice lead, NHS Grampian, Aberdeen, UK; ' ${ }^{\circ}$ director of public health, Public Health Gibraltar, Gibraltar, UK and head of Postgraduate Medical School, University of Gibraltar, UK
With experience, many are realising the impact of 'best supportive care' on actual mortality levels in COVID-19 infection. As many of us face the surge in cases, and others face accepting COVID-19 as part of our medical take for some time to come, it is useful to remember how much can be done to affect the trajectory of illness, even without a 'magic bullet'.

\section{Vigilance}

As evidence accrues, it is becoming clearer that early intervention matters. Several studies have reported that late presentation is directly associated with increased mortality.

Zhou et al followed up 191 patients from admission to discharge or death. Of the 54 patients who died, the average time to hospital admission was 11 days. Over $70 \%$ of those presenting who eventually died arrived at hospital in a critical condition. High respiratory rate $(R R>24)$ at presentation was more common in those who did not survive versus those that did (but it is worth noting was not present in $37 \%$ of cases with fatal outcome). CURB-65 scores were rarely more than 1 in the surviving group, but 2 or more in $71 \%$ of non-survivors. ${ }^{3}$

A similar finding was found in South Korea. ${ }^{4}$ Despite South Korea maintaining a lower than average case fatality rate (CFR), they report that $15 \%$ of fatalities occurred while the patient was still at home. Again, average time to presentation in those who did not survive was 10 days.

Based on initial evidence that emerged early on in the outbreak, there was the suggestion that the ARDS associated with severe COVID-19 infection presents in the second week of the illness. As such, one can attribute the delayed presentation of 10-11 days simply to the timing of the ARDS in COVID-19. However, the initial data from the US would go against such an assumption. 5 Detailed data on the first 24 cases admitted to the Critical Care Unit (CCU) in the Washington State area reveal that patients can present with severe ARDS early on in the illness. Out of the 24 patients admitted to the CCU, 9 patients had a history between $1-5$ days in duration. Of those 9 patients, 8 died.

The Seattle Critical Care Cohort mentioned above also highlighted the critical nature of patients presenting to hospital, with 11 out of 25 patients being ventilated on day 1 of admission. ${ }^{5}$

\section{Early intervention}

In the absence of medication that can alter the trajectory of the illness, it is easier to maintain the stance of recommending 
'self-isolation' and 'self-care'. For most patients, it appears that COVID-19 is a self-limiting viral infection. ${ }^{6}$ Typically it presents with upper respiratory tract infection (URTI) symptoms, or some other viral constellation. Patients are often febrile and feel washed-out, with symptoms not too dissimilar to those seen in flu. COVID-19 typically becomes an issue when the patient becomes short of breath. Shortness of breath is not a symptom of an URTI. It invariably (bar the occasional anxious patient) heralds the onset of viral pneumonia, pneumonitis or ARDS. It is a red flag symptom, and requires further assessment.?

There are other worrying symptoms to be mindful of. Pyrexia lasting more than 3 days seldom bodes well in infectious diseases. Pyrexia remaining at day seven is even more concerning. Such patients will likely have a significant inflammatory response, and likely lung pathology. There are also the usual signs of sepsis and the risk of secondary bacterial infection that may be difficult to distinguish from the novel coronavirus without some pointed questions

And it is worth asking the questions. Most of the lung injury associated with progressive COVID-19 relates to inflammation. ${ }^{8,9}$ It seems that viral load falls off quite quickly in most, and then in some there is a hyperimmune reaction leading to destruction of lung tissue, with or without a systemic inflammatory response syndrome (SIRS). The subsequent hypoxia is itself pro-inflammatory and if undetected and uncorrected drives the inflammatory process forward. ${ }^{10}$ Hence, time to supplemental oxygen in a hypoxic patient with COVID-19 may well affect the trajectory of the disease.

Hypoxia under physical stress is even more pro-inflammatory. ${ }^{11}$ Allowing people with acute inflammatory lung pathology to rest is helpful. Removing them from the obligations of day-to-day life - caring for others, preparing food, household chores, etc - is useful for recovery. There are a great number of interventions that constitutes 'best supportive care', even before we move into the realm of organ support (Table 1).

Indeed, Sun et al reports on how Jiangsu Province managed to lower its CCU admission rate and with it their CFR. ${ }^{12}$ Primarily, they used high levels of vigilance (including prognostic markers) to identify patients who were likely to deteriorate. Such patients were admitted and monitored for deterioration. Early supplemental oxygen was described as one of the key interventions preventing patients requiring intubation. With a severe case rate of $10 \%$ and a mechanical ventilation rate of less than $1 \%$, something was working. Given the quite dramatic fall in CFR from Wuhan (10\%) to the rest of China $(0.6 \%)$, it is worth considering the merits of vigilance and early intervention.

It is also worth noting the Singapore response to the outbreak and the subsequent remarkably low CFR. All patients with suspected or confirmed COVID-19 were assessed in hospital and most were admitted. ${ }^{13}$

\section{Discussion}

In many places - perhaps more particularly in places with substantive public healthcare systems such as the NHS - there is often a default position of avoiding admission and facilitating rapid discharge of patients. ${ }^{14}$ It is often a necessary stance to take as the demands on us increase..$^{15}$ In current times, with this new foe, the mindset of avoiding admissions needs to be tempered by the knowledge of the importance of early intervention in this new disease.
Table 1. Hospital interventions likely to improve outcome in patients with severe COVID-19 infection

\begin{tabular}{ll} 
Supportive care & Rest \\
& Appropriate hydration \\
& Nutritional support \\
& VTE prophylaxis \\
& Pain management - improving respiration \\
& Management of comorbidities \\
& COPD/asthma - nebulisers and steroids \\
& Heart failure management \\
& Fluid balance in renal failure \\
& Titration of blood pressure medication \\
& Adjusting immunosuppressive medication \\
& Safe glycemic control \\
& Early identification of secondary bacterial \\
& infection/sepsis \\
Early & Identification of 'COVID-19' mimickers \\
identification & (eg urosepsis, heart failure, pulmonary \\
& embolism, CAP, HAP) \\
& Early identification of hypoxia \\
& Early identification of secondary \\
& pulmonary embolism \\
& Early identification of type-2 \\
& myocardial infarction \\
& Escalation plan discussions \\
& Patients requiring CCU care \\
\hline &
\end{tabular}

$\mathrm{CAP}=$ community-acquired pneumonia; $\mathrm{COPD}=$ chronic obstructive airways disease; HAP = hospital-acquired pneumonia; $\mathrm{VTE}=$ venous thromboembolism.

An overwhelmed healthcare system does poorly, mainly due to the loss of the usual checks and balances that prevent people deteriorating in the community, presenting late and then requiring heroic measures to give them a fighting chance. If the curve has been flattened - if we have dampened the surge - and the contingencies have freed up some space, then it would be wise to use such resource to proactively identify patients at risk and assess them regularly, while maintaining a lower threshold for admission than perhaps we are used to. Intervening early is likely to use less resources and save more lives.

The alternative is to allow patients to regulate themselves. Unless the patient has a medical degree and an oxygen saturation monitor at home, they are not in a position to determine whether they should break the 'stay at home' mantra that has gripped much of the world. Perhaps without such pressure to stay at home, people could use their normal (non-pandemic) judgement to determine when to seek medical help. They are disadvantaged further by the lack of actual physical contact with their community doctor. Again, assessing a patient's clinical status over the phone, with an infection we have never seen before, is asking a lot of both clinician and patient.

The battle for lives will likely be won in the community. It is the community doctors, the paramedics and the emergency department staff who will shift the balance and save most lives. For most it will be, clear, sound triage systems and clear clinical guidelines that will determine the mortality rate probably much more than the total number of ventilators available. 


\section{References}

1 Lake MA. What we know so far: COVID-19 current clinical knowledge and research. Clin Med 2020;20:124-7.

2 Greenberg N, Docherty M, Gnanapragasam S, Wessely S. Managing mental health challenges faced by healthcare workers during covid-19 pandemic. BMJ 2020;368:m1211.

3 Zhou F, Yu T, Du R et al. Clinical course and risk factors for mortality of adult inpatients with COVID-19 in Wuhan, China: a retrospective cohort study. Lancet 2020:395:1054-62.

4 Korean Society of Infectious Diseases and Korea Centers for Disease Control and Prevention. Analysis on 54 mortality cases of coronavirus disease 2019 in the Republic of Korea from January 19 to March 10, 2020. J Korean Med Sci 2020;35:e132

5 Bhatraju PK. Covid-19 in critically ill patients in the Seattle region - case series. N Engl ] Med 2020, in press (DOI: 10.1056/ NEJMoa2004500).

6 Xie M, Chen Q. Insight into 2019 novel coronavirus - an updated interim review and lessons from SARS-CoV and MERS-CoV. Int ] Infect Dis 2020;94:119-24.

7 Greenhalgh T, Koh GCH, Car J. Covid-19: a remote assessment in primary care. BMJ 2020; 368:m1182.

8 Channappanavar R, Perlman S. Pathogenic human coronavirus infections: causes and consequences of cytokine storm and immunopathology. Semin Immunopathol 2017;39:529-39.

9 Zhang $\mathrm{W}$, Zhao $\mathrm{Y}$, Zhang $\mathrm{F}$ et al. The use of anti-inflammatory drugs in the treatment of people with severe coronavirus disease
2019 (COVID-19): the perspectives of clinical immunologists from China. Clin Immunology 2020;214:108393.

10 Eltzschig HK, Carmeliet P. Hypoxia and inflammation. N Engl J Med 2011:17:364:656-65.

11 Kammerer T, Faihs V, Hulde $\mathrm{N}$ et al. Hypoxic-inflammatory responses under acute hypoxia: in vitro experiments and prospective observational expedition trial. Int ] $\mathrm{Mol} \mathrm{SCi}$ 2020;21:1034.

12 Sun Q, Qiu H, Huang M, Yang Y. Lower mortality of COVID-19 by early recognition and intervention: experience from Jiangsu Province. Ann Intensive Care 2020;18:10:33.

13 Wong JEL, Leo YS, Tan CC. COVID-19 in Singapore - current experience: critical global issues that require attention and action. JAMA, 2020, in press (DOI: 10.1001/jama.2020.2467).

14 Bentley JA, Thakore S, Morrison W, Wang W. Emergency Department redirection to primary care: a prospective evaluation of practice. Scott Med J 2017;62:2-10.

15 Pope I, Burn H, Ismail SA, Harris T, McCoy D. A qualitative study exploring the factors influencing admission to hospital from the emergency department. BMJ Open 2017;7:e011543.

Address for correspondence: Dr Daniel Goyal, Acute General Medicine, St Bernard's Hospital, Harbour Views Road, Gibraltar GX11 1AA, Gibraltar.

Email: daniel.goyal@gha.gi

\section{Consent and confidentiality in genomic medicine}

\section{Genetic or genomic tests are increasingly used in everyday medical practice. Every clinical field will encounter such tests to a greater or lesser extent.}

Published in 2019, this third edition by the Joint Committee on Genomics in Medicine provides updated guidance on the use of genetic and genomic information in the clinic. Health professionals from all areas of medicine need to know and understand how consent and confidentiality issues may arise, and to understand the potential ways in which the use of genomic tests may change the nature of the relationship between healthcare professionals and patients.

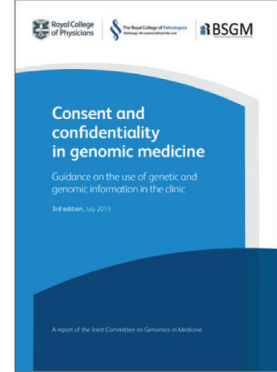

Download the guidance at: www.rcplondon.ac.uk/consent-confidentiality-genomic-medicine 